\title{
Expression of the green fluorescent protein in Paramecium tetraurelia
}

\author{
Karin Hauser ${ }^{\mathrm{a}}$, W. John Haynes ${ }^{\mathrm{b}}$, Ching Kung ${ }^{\mathrm{b}}$, Helmut Plattner ${ }^{\mathrm{a}}$ and Roland Kissmehl $\left.{ }^{1}\right)^{\mathrm{ab}}$ \\ ${ }^{a}$ Faculty of Biology, University of Konstanz, Konstanz/Germany \\ b Laboratory of Molecular Biology, University of Wisconsin-Madison, Wisconsin/USA
}

Received July 27, 1999

Received in revised version September 23, 1999

Accepted October 26, 1999

GFP - expression - Paramecium - transformation

In this paper we describe the expression of green fluoresent protein (GFP) as a reporter in vivo to monitor transformation in Paramecium cells. This is not trivial because of the limited number of strong promoters available for heterologous expression and the very high AT content of the genomic DNA, the consequence of which is a very aberrant codon usage. Taking into account differences in codon usage we selected and modified the original GFP open reading frame (ORF) from Aequorea victoria and placed the altered ORF into the Paramecium expression vector pPXV. Injection of the linearized plasmid into the macronucleus resulted in a cytoplasmic fluorescence signal in the clonal descendants, which was proportional to the number of copies injected. Southern hybridization indicated the establishment and replication of the plasmid during vegetative growth. Expression was also monitored by Northern and Western analysis. The results indicate that the modified GFP can be used in Paramecium as a reporter for transformation as an alternative to selection with antibiotics and that it may also be used to construct and localize fusion proteins.

Abbreviations. APH-3'-II Aminoglycoside 3'-phosphotransferase-II/neor gene. - CAM Calmodulin from wild-type Paramecium. - GFP Green fluorescent protein. - GFPmutl Green fluorescent protein with F64L and S65T substitutions. - ORF Open reading frame. - pPXV Paramecium expression vector. - pPXV-GFP Paramecium expression vector with the open reading frame of green fluorescent protein. - pPXV-NEO Paramecium expression vector with the open reading frame of aminoglycoside 3'-phosphotransferase-II/neo' gene. - Wt Wild-type.

1) Dr. Roland Kissmehl, Laboratory of Molecular Biology, University of Wisconsin-Madison, 1525 Linden Drive, Rm 305, Madison, Wisconsin 53706/USA, e-mail: rakissmehl@facstaff.wisc.edu, Fax: + +6082624570.

\section{Introduction}

Paramecium tetraurelia is a unicellular organism which is used as a model system for many basic cellular processes. Many mutants are available and its size of about $100 \mu \mathrm{m}$ allows for easy manipulation and observation. It can be transformed not only by microinjection, but also by electroporation (Fraga et al., 1998; Boileau et al., 1999) or by bioballistic particle bombardment (Boileau et al., 1999). So far, the only method of selecting transformed wild-type Paramecium cells is by an antibiotic resistance marker (Haynes et al., 1995). This marker has the disadvantage that successful transformation can be monitored only after several days when transformed cells have grown for five or six generations to produce enough descendants which subsequently can be checked for drug resistance.

An alternative is to positively select cells with reporter systems like the green fluorescent protein (GFP) from Aequorea victoria (reviewed by Tsien, 1998). The GFP gene has been cloned and expressed successfully in many organisms ranging from E. coli to mammals and plants (Prasher et al., 1992; Chalfie et al., 1994; Inouye and Tsuji, 1994; Cubitt et al., 1995). While the entire 27-kDa monomer consisting of 238 amino acids is required for fluorescence, the active chromophore of GFP is only a tripeptide found in the middle of the protein (Cody et al., 1993). Upon excitation with blue or UVlight this tripeptide undergoes a cyclization reaction which does not appear to be enzymatic but requires molecular oxygen and causes the protein to emit bright green light (Heim et al., 1994; Inouye and Tsuji, 1994).

Although there are many commercially available chromophore variants with specific amino acid substitutions that enhance fluorescence and alter emission spectra of GFP (Heim et al., 1995; Tsien, 1998), most of them have had a substantial number of silent mutations introduced to efficiently express the jellyfish gene in mammalian and plant cells. Since the codon usage in Aequorea victoria is closer to that in ciliates, wild-type GFP was selected for expression in Paramecium. Two amino acid substitutions were made at positions F64L and S65T because earlier studies of the gene showed that 
these substitutions produce a higher extinction coefficient, cyclize the tripeptide more efficiently to form the chromophore, and subsequently cause the protein, referred to as GFPmut1, to fluoresce up to 35-fold more intensely than wildtype GFP when excited at $488 \mathrm{~nm}$ (Cormack et al., 1996; Yang et al., 1996). In addition, a TGA stop codon was introduced at the 3 '-end of the GFP ORF because the original TAA found in the Aequorin gene codes for glutamine in Paramecium (Preer et al., 1985; Prescott, 1994).

To our knowledge at the time of construction of this reporter, the only available vector for heterologous expression in Paramecium was pPXV (Haynes et al., 1995). Once introduced into pPXV, the GFP ORF will be flanked by cisacting transcriptional elements from the endogenous calmodulin (CAM) gene, and when the plasmid is linearized telomeric sequences from Tetrahymena help to stabilize the injected fragment in the macronucleus (Gilley et al., 1988; Kanabrocki et al., 1991; Haynes et al., 1995).

Here, we now describe the successful adaption and expression of GFP in Paramecium tetraurelia.

\section{Materials and methods}

\section{Stocks and cell culturing}

P. tetraurelia, nd6/nd6 with 51S background (Lefort-Tran et al., 1981) and wild-type strain $7 \mathrm{~S}$ (Sonneborn, 1975), was cultured at $22^{\circ} \mathrm{C}$ in a growth medium of buffered wheat-grass extract inoculated with Enterobacter aerogenes (Sonneborn, 1970). Single cells were isolated and grown for more than twenty fissions before each clone was starved to induce autogamy (Berger, 1986). Autogamy was monitored by fluorescence microscopy after fixing and staining with DAPI $\left(4^{\prime}, 6-\right.$ diamidino-2-phenylindole) (Haynes et al., 1995). Autogamous cells were then fed and used for transformation between the third and sixth post-autogamous divisions.

\section{Construction of PPXV-GFP}

To amplify and modify wild-type GFP, several PCR steps were performed using plasmid pGFP-C1 (EMBL accession number U19280, Clontech Laboratories, Inc. Palo Alto, CA, USA) as a template and primer pair combinations as follows: For the first primer pair, 5'-oligonucleotide 1 at position 580-598 (numbers refer to U19280) 5'CCGTCAGATCCGCTAGCGC3', which has an internal Nhe I restriction site (italic), was paired with $3^{\prime}$-oligonucleotide 2 (pos. 816-790) 5'AACACCATAAGTTAACGTAGTGACAAG3', which carries nucleotides for a F64 $\mathrm{L}$ and S65T substitution (bold) and an additional Hinc II restriction site (underlined). For the second primer pair, 5'-oligonucleotide 3 (pos. 790-816) 5'CTTGTCACTACGTTAACTTATGGTGTT3', which is the exact complement of $3^{\prime}$-oligonucleotide 2, was combined with 3 '-oligonucleotide 4 (pos. 1352-1318) 5'GCTTGAGCTCGAGATCTGAGGCCTCACTTGTATAG3', which contains nucleotide exchanges (bold characters) for an additional Stu I restriction site (underlined) and a stop codon in the reverse sense strand (double underlined, complementary). The italic characters indicate the Xho I restriction site used for cloning. PCRs were performed using $2.5 \mathrm{U}$ cloned Pfu DNA polymerase (Stratagene, La Jolla, CA, USA). Cycling started with an initial denaturation step for 5 min at $95^{\circ} \mathrm{C}$, followed by 35 cycles with $45 \mathrm{~s} 95^{\circ} \mathrm{C}, 30 \mathrm{~s} 56^{\circ} \mathrm{C}, 3 \mathrm{~min}$ $30 \mathrm{~s} 72^{\circ} \mathrm{C}$ and a final extension of $20 \mathrm{~min}$ at $72^{\circ} \mathrm{C}$. Amplification products were purified and either reused as template for PCR or cut by restriction endonucleases and cloned into pPXV (Haynes et al., 1995). The construct was then confirmed by sequencing the complete GFPmut1 ORF.

For transformation pPXV-GFP was cleaved with Sfi I to release a linearized fragment containing the calmodulin promoter, GFP ORF, and calmodulin $3^{\prime}$ untranslated region flanked by vector and telomeric sequences.

\section{Transformation by microinjection}

Serial dilutions were made of Sfi I-linearized pPXV-GFP $(0.58 \mathrm{ng} / \mu \mathrm{l}-$ $5.8 \mu \mathrm{g} / \mu \mathrm{l})$. Five to ten picoliters of each dilution were microinjected into the macronucleus as previously described (Haynes et al., 1995; Haynes et al., 1996). Injected or non-injected control cells were isolated and grown for at least three days at room temperature in bacterized wheat grass medium diluted $1: 1$ with double-distilled water, before they were analyzed for GFP expression.

\section{Transformation by bioballistic particle bombardment}

Cells were washed twice in Hepes buffer ( $5 \mathrm{mM}$ Hepes- $\mathrm{HCl}, 5 \mathrm{mM}$ $\mathrm{KCl}, 1 \mathrm{mM} \mathrm{CaCl} 2, \mathrm{pH} 7.2)$, before 0.5 -ml aliquots (2500-7500 cells) were bombarded with gold particles $(1.6 \mu \mathrm{m}$ diameter $)$ coated with $2-$ $4 \times 10^{4}$ Sfi I-linearized pPXV-GFP molecules as previously described (Boileau et al., 1999). Cells were immediately transferred into bacterized wheat grass media, cultured for 3 days and then examined for GFP expression.

\section{Monitoring of GFPmut1 fluorescence}

Transformants were analyzed with a Nikon Diaphot 300 inverted microscope using a Plan $20 \times$ DL, 0.5 NA objective and a High Q Endow GFP longpass emission filter set (Chroma Technology Corp., Brattleboro, VT, USA). Images were projected for $2 \mathrm{~s}$ onto a CH250 slow scan, cooled CCD camera (Photometrics Ltd., Tucson, AZ, USA). Digitized 16 bit images at $0.5 \mu \mathrm{m} /$ pixel were collected using Metamorph imaging software (Universal Imaging Corp., Westchester, PA, USA). In some cases images were rescaled to a 256 greyscale using NIH image software. 20 independent cytosolic measurements of 25 square pixels were averaged (avoiding vacuoles, nuclei, or the oral region) and background values obtained from areas with no cells were subtracted.

\section{Analyses of DNA, RNA and protein from clonal descendants of pPXV-GFP injected cells}

Wild-type transgenic $7 \mathrm{~S}$ cells were grown after transformation for about $18-20$ fissions to obtain clones containing about 400000 cells in $325 \mathrm{ml}$ bacterized wheat grass media. Cultures were checked for GFP fluorescence before harvest. Cells were washed twice in Pipes-KOH buffer ( $5 \mathrm{mM}$ Pipes, $1 \mathrm{mM} \mathrm{KCl,} 0.1 \mathrm{mM} \mathrm{CaCl}_{2}, \mathrm{pH} 7.0$ ) and kept for 4 hours in the same buffer to allow complete digestion of the bacteria present in food vacuoles. Genomic DNA was prepared from about 70000 cells according to Godiska et al. (1987). For Southern blot analysis $10 \mu \mathrm{g}$ of genomic DNA were digested with BamH I and blotted onto Qiabrane (Qiagen, Hilden, Germany). Blots were hybridized with a probe obtained by PCR using 5 '-oligonucleotide 1 and 3 -oligonucleotide 4 as primers and pGFP-C1 as a template (see above). The probe was labeled with $\left[\alpha-{ }^{32} \mathrm{P}\right] \mathrm{dCTP}$. Blots were hybridized at $42{ }^{\circ} \mathrm{C}$ in $50 \%$ formamide according to Sambrook et al. (1989). Total RNA was prepared from $\sim 130000$ cells using the RNAgents total RNA Isolation System from Promega (Madison, WI, USA). For Northern blot analysis $20 \mu \mathrm{g}$ of total RNA were loaded per lane and blotted onto Qiabrane. Probe and hybridizing conditions were the same as for Southern analysis. For Western analysis, a $100000 \mathrm{~g}$ supernatant and pellet fraction was prepared from about 200000 cells and processed according to Kissmehl et al. (1998) using a monoclonal antibody against GFP (Clontech Laboratories GmbH, Heidelberg, Germany).

For controls genomic DNA, total RNA and protein fractions were also analyzed from non-transformed wild-type $7 \mathrm{~S}$ cells.

\section{Results}

\section{Choice of the green fluorescent protein}

Like other ciliates $P$. tetraurelia employs a divergent genetic code by using the universal stop codons TAA and TAG for glutamine (Preer et al., 1985; Prescott, 1994). Together with the ciliate-specific codon usage (Martindale, 1989) this should 
be considered when an appropriate GFP gene has to be selected for heterologous expression in Paramecium. In a preliminary study, we estimated the preferred codons in Paramecium by combining data from a previous evaluation of surface variant antigens (Martindale, 1989) with those from our own analysis of more recently published Paramecium genes, like the plasma membrane $\mathrm{Ca}^{2+}$-ATPase (Elwess and Van Houten, 1997), the phosphoprotein PP63 (Hauser et al., 1997), centrin (Madeddu et al., 1995), and dihydrofolate reductase-thymidylate synthase (Schlichtherle et al., 1996). When used in a subsequent comparison with codons found in the various commercially available GFP variants, only the original GFP wild-type gene from Aequorea victoria revealed an almost identical codon usage as Paramecium (data not shown), which therefore was selected for modification and expression.

\section{Construction of pPXV-GFP}

In the first step, a DNA fragment which contains the complete coding region of wild-type GFP with an F64L and S65T substitution in the chromophore (previously described as GFPmut1; Cormack et al., 1996) and a functional TGA stop codon at the end of the open reading frame was amplified by PCR (Fig. 1a). In a next step, this DNA molecule was digested with Nhe I and Xho I and ligated into Spe I/Xho I doubledigested $\mathrm{pPXV}$, which represents the only expression vector available for P. tetraurelia (Haynes et al., 1995). Since Spe I and Nhe I produce compatible cohesive ends this cloning step

\section{a}

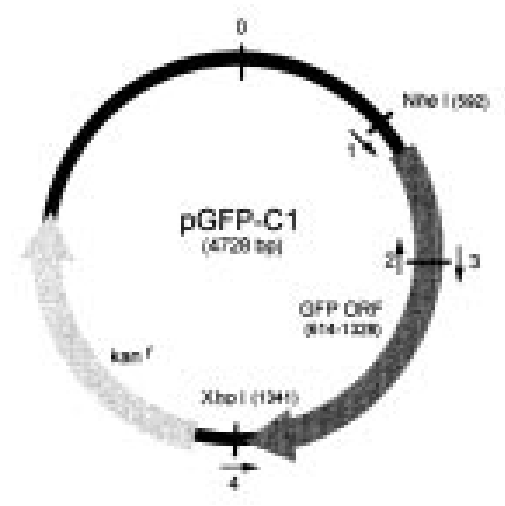

b

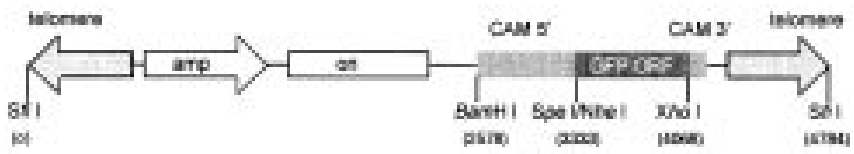

Fig. 1. Construction of pPXV-GFP. (a) PCR strategy for mutation of wt GFP into GFPmut1. With pGFP-C1 as a template two PCRs were performed using either oligonucleotides 1 and 2 or olignucleotides 3 and 4 as primers. The resulting two PCR products were purified and used as templates for a subsequent PCR step with oligonucleotides 1 and 4 as primers. This resulted in a DNA fragment containing the complete coding region for wt GFP with mutations in the chromophore (F64L and S65T substitutions) and a TGA stop codon at the end of the ORF. (b) Schematic map of Sfi I-digested pPXV-GFP. Nhe I (Spe I) and Xho I restriction sites were used for cloning into the $P$. tetraurelia expression vector $\mathrm{pPXV}$. The new construct is named pPXV-GFP. (amp ampicillin resistance, $\mathrm{kan}^{\mathrm{r}}$ kanamycin resistance, ori origin of replication, CAM 5' Paramecium calmodulin promoter, CAM 3' Paramecium calmodulin termination sequences, GFP ORF coding region of GFPmut1, telomer Tetrahymena telomer sequences) allows the modified GFP gene to be located behind the constitutive promoter of the $P$. tetraurelia calmodulin (CAM) gene, which was already demonstrated to ensure successful expression of homologous and heterologous genes in Paramecium (Haynes et al., 1995, Haynes et al., 1996). The new construct was referred to as pPXV-GFP (Fig. 1b).

\section{Expression of GFPmut 1 in $\boldsymbol{P}_{\text {. }}$ tetraurelia}

In order to create a DNA fragment, in which telomeric sequences were exposed on both ends of the gene-bearing fragment, pPXV-GFP was linearized by Sfi I (Fig. 1b). When $5 \times 10^{6}$ to $1 \times 10^{7}$ copies in a volume of approximately $5-10 \mathrm{pl}$ of linearized pPXV-GFP molecules were injected into the macronucleus of $P$. tetraurelia cells, GFP fluorescence could easily be observed already $12 \mathrm{~h}$ after injection, although clonal descendants were usually analyzed at a later stage, mainly after $5-7$ fissions. Such a GFP-derived fluorescent signal is shown in Figure 2a. With the exception of several vacuoles and several bright spots the signal is generally distributed throughout the cell including the macronucleus. Since descendants from noninjected control cells (Fig. 2a) or from those injected with only Sfi I linearized pPXV vector sequence (data not shown) do not show any fluorescence above background, this strongly indicates successful expression of GFPmut1. When clonal descendants were allowed to undergo autogamy ( $\geq 20$ fissions), the GFP fluorescence signal was lost (data not shown).

\section{Fluorescence of GFPmut1 depends on copy number}

In order to evaluate the critical copy number for detectable GFPmut1 fluorescence, cells were injected with decreasing copy numbers of linearized plasmids $\left(10^{6}, 10^{5}\right.$, or $10^{4}$ copies $\left./ \mathrm{pl}\right)$ and compared to a cell from a non-injected control clone. A strong dose-dependent decrease in cytoplasmic GFP fluorescence was observed (data not shown) which has been corroborated in a semi-quantitative analysis (Fig. 2b). Microinjection of less than $10^{4}$ copies/pl did not result in any signal above background (data not shown). The relative fluorescence signal at the critical concentration of $10^{4}$ copies/pl was analyzed in more detail (Fig. 2c). Data were retrieved from a picture (not shown), in which cells from several independently injected clones were placed together and compared with a noninjected control cell. Again, semi-quantitative evaluation was based on the fluorescence signal only from the cytosolic compartment, although fluorescence was also observed in the macronucleus (Fig. 2a). Furthermore, we also avoided measurements in other (visible) subcellular compartments, such as vacuoles or the oral region. At this concentration, values obtained from all transformants still demonstrated a GFPderived fluorescence signal above background (Fig. 2c).

\section{GFPmut 1-derived fluorescence in bioballistically transformed cells}

Descendants of cells transformed with Sfi I-digested pPXVGFP by bioballistic particle bombardment showed GFPmut1 fluorescence, well above the critical concentration analyzed in Figure 2c for microinjected cells (data not shown). However, transformation efficiency was below $0.1 \%$ due to the relative high amount of GFPmut1 molecules necessary for monitoring fluorescence in Paramecium (see also "Discussion" section). 


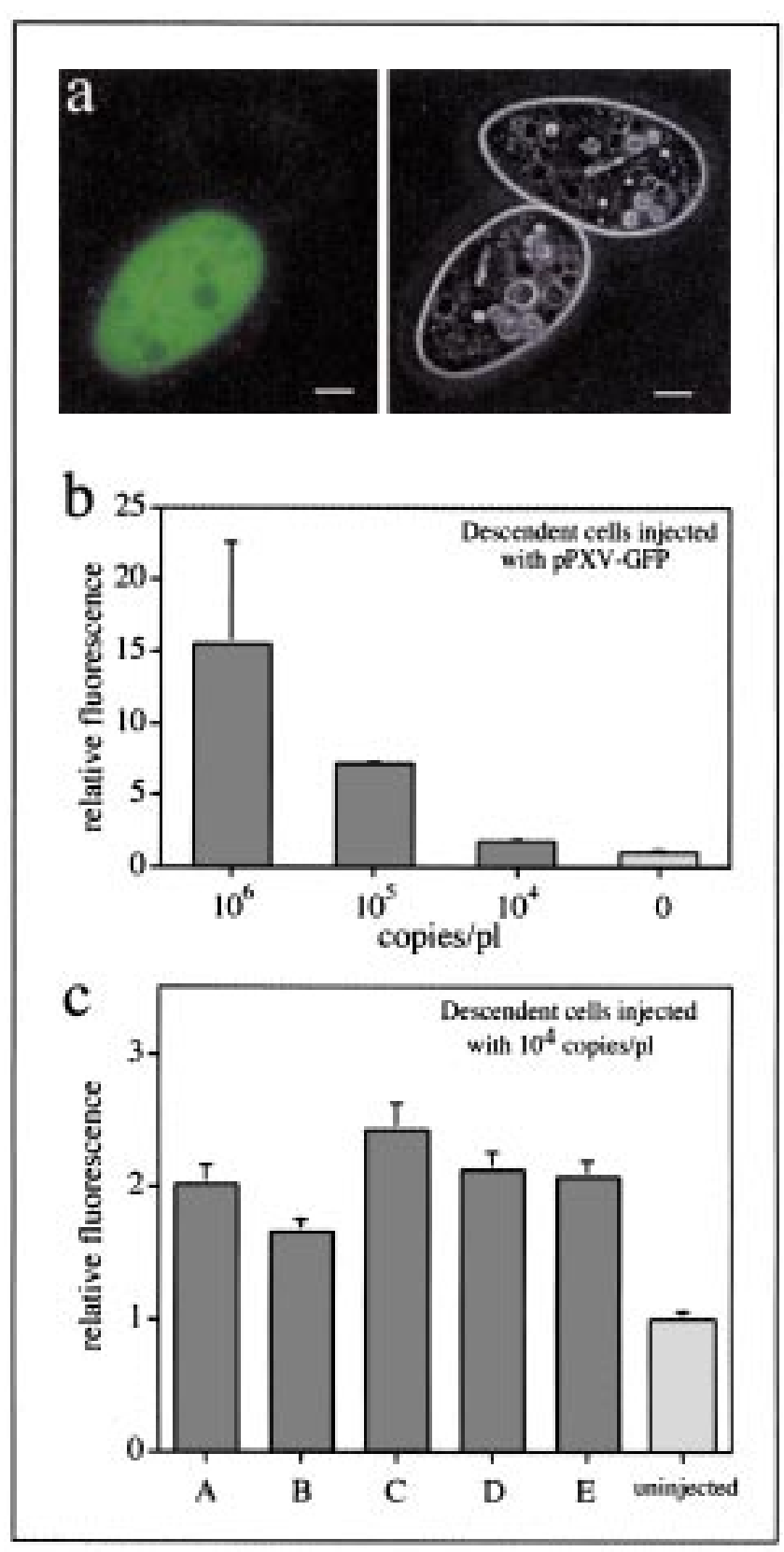

Fig. 2. Cytoplasmic fluorescence in Paramecium cells after injection of pPXV-GFP. (a) right panel, the fluorescent signal from a descendent cell three days after injection with Sfi I-digested pPXV-GFP $\left(10^{6}\right.$ copies/pl) compared with a non-injected cell strongly indicates GFP expression. With the exception of several vacuoles and several bright spots the signal is generally distributed throughout the cell including the macronucleus. The corresponding phase micrograph is shown in the left panel. Bars $=20 \mu \mathrm{m}$. (b) The relative cytoplasmic fluorescent signal from each of four cells injected with $10^{6}, 10^{5}, 10^{4}$ and 0 copies/pl respectively, was estimated by averaging 20 independent cytosolic measurements (mean value of 25 square pixels) followed by subtraction of a mean background value (off-cell) and normalizing to the fluorescence value obtained from the non-injected control cell. (c) The signal from descendent cells from several independent injections of pPXV-GFP at the lowest concentration detected $\left(10^{4} \mathrm{copies} / \mathrm{pl} ; \mathrm{A}-\mathrm{E}\right)$ are compared to a single uninjected cell by relative fluorescence. Each data point is a mean $\pm S D(n=20)$.

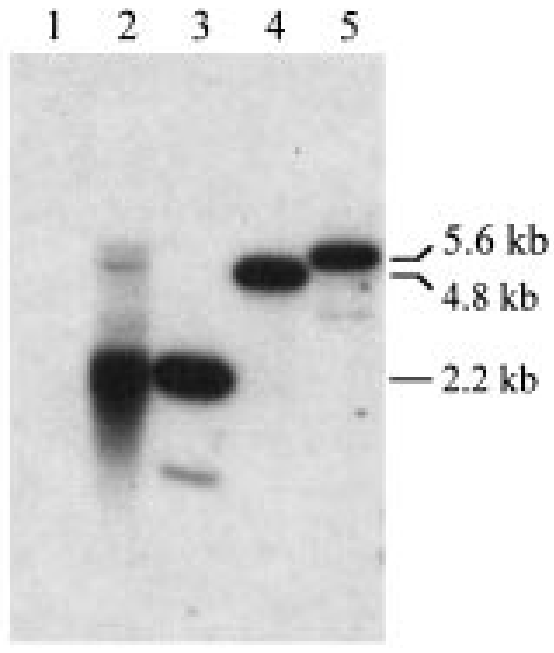

Fig. 3. Southern hybridization analysis of pPXV-GFP transformants. For Southern hybridizations $10 \mu \mathrm{g}$ of BamH I-digested Paramecium DNA from either non-injected control cells (lane 1) or from clones injected with 5-10 $\times 10^{6}$ copies of Sfi I-linearized pPXV-GFP (lane 2) were probed with the complete ORF of GFPmut1 and compared in control hybridizations with $200 \mathrm{pg}$ of pPXV-GFP, double-digested with Sfi I/BamH I (lane 3) or single-digested with either Sfi I (lane 4) or BamH I (lane 5).

\section{Analysis of the injected transgene GFPmut 1 on DNA, RNA and protein level}

Descendants from pPXV-GFP injected cells $\left(5-10 \times 10^{6}\right.$ copies of Sfi I-linearized plasmid) or from non-injected control cells were grown for almost twenty generations to allow preparation of sufficient DNA, total RNA and protein.

Presence or absence of GFPmut1-specific DNA sequences in injected and non-injected clones was investigated by Southern hybridizations in which $10 \mu \mathrm{g}$ of isolated total DNA was cleaved with BamH I before being used as a sample (Fig. 3). BamH I cuts Sfi I digested pPXV-GFP into two fragments of $2206 \mathrm{bp}$ and $2578 \mathrm{bp}$ (lane 3). The $2206 \mathrm{bp}$ fragment contains the complete GFPmut1 ORF, which, therefore, was recognized by a probe specific for GFPmut1 (lane 2). This band had a fuzzy appearance and was similar to diffuse signals seen in association with telomere elongation (McCormick-Graham et al., 1997). However, there were also two minor signals at $5.6 \mathrm{~kb}$ and $4.8 \mathrm{~kb}$ which were not expected, but which might result from incomplete digestion with Sfi I prior to transformation (lane 4) or with BamH I after transformation (lane 5). Specificity was guaranteed since no signal could be detected in DNA from non-injected control cells (lane 1).

Expression of GFP was also monitored by Northern and Western analysis, in which a mRNA transcript of $\sim 840$ bp and a newly synthesized soluble protein of about $27 \mathrm{kDa}$ were found exclusively in GFPmut1-transformed cells (data not shown). This also reflects successful expression of GFP in Paramecium tetraurelia.

\section{Discussion}

The only marker previously available for transformation of wild-type cells of Paramecium tetraurelia was based on the heterologous expression of the bacterial antibiotic, aminoglycoside 3'-phosphotransferase-II gene (APH-3'-II or neo ${ }^{r}$, 
which, upon microinjection (Haynes et al., 1995) or transformations en masse (Boileau et al., 1999), confers resistance to the drug G-418. However, this antibiotic selection has the disadvantage that unequivocal transformations can be ascertained only after several days of incubation in the selection medium under conditions that probably are not optimal for growth, even for transformed cells. Therefore, there was a need for a marker that could be expressed and detected rapidly at the level of a single cell and if possible, without significantly altering the physiological conditions.

Preliminary studies, where recombinant GFP molecules were microinjected into the cytoplasm, showed that the physiological conditions prevailing in the Paramecium cell allowed for the protein to fluoresce (data not shown). While the common commercial varieties of the GFP gene have been adapted for optimal expression in mammalian cells (Haas et al., 1996), the original gene from Aequorea victoria has a codon usage similar to that observed in Paramecium. Injection of the expression plasmid, with the three modifications in the GFP ORF, into the macronucleus resulted in a clear green fluorescent signal from the cytoplasm, when excited with the appropriate wavelength. This signal could clearly be distinguished from autofluorescence in control cells (Fig. 2), even when the signal was derived from clonal descendants after 18 to 20 fissions.

The intensity of GFP fluorescence strongly depends on the number of copies of pPXV-GFP injected (Fig. $2 b-c)$. The lowest copy number which resulted in a visible fluorescence signal above autofluorescence, was about $5-10 \times 10^{4}$ copies injected per cell which is similar to that required for antibiotic resistance with pPXV-NEO. However, the fluorescent signal from GFP is directly proportional to the number of molecules expressed, which therefore can be used to approximate the amount of protein produced by the calmodulin cassette.

Since it is difficult to assess the number of plasmids retained in the macronucleus after injection and because there is a tendency to loose plasmid (Haynes et al., 1996) we will make the estimates derived from the number of injected plasmids, which might overestimate their actual concentration at later clonal stages. Previous studies have shown that EGFP (GFPmut1) has a half-life of more than $24 \mathrm{~h}$ (Li et al., 1998) and that the mutations in the chromophore reduce photobleaching (Patterson et al., 1997). Taking advantage of these characteristics, Patterson and co-workers showed, in a variety of mammalian cell types, that a cytoplasmic concentration around $200 \mathrm{nM}$ was needed for detection above the background autofluorescence (Patterson et al., 1997). Although the autofluorescence from Paramecium is significantly higher than in mammalian cells, we can assume the same minimum concentration of $200 \mathrm{nM}$ GFPmut1 is required. Together with an estimation of a cell volume at about $\sim 75 \mathrm{pl}$ for $P$. tetraurelia (Erxleben et al., 1997), about $9 \times 10^{6}$ GFP molecules must be present in a cell. This number is $10^{2}$ to $10^{3}$-fold higher than that needed for mammalian cells (Patterson et al., 1997; Tsien, 1998), but this is consistent with their smaller size. While the requirement for such extraordinary amounts of protein on the one hand may explain the low level of detectable bioballistic-based transformation, on the other hand this demonstrates the effectiveness of the calmodulin cassette for heterologous expression. Using the estimations of the minimum number of protein molecules needed to detect a fluorescent signal above background and the maximum number of plasmids that could be retained in the macronu- cleus, a number of $\sim 10^{2}$ protein molecules for every injected plasmid is derived. This is actually much lower than an estimation of native expression levels for calmodulin $\left(>10^{6}\right.$ protein molecules per endogenous gene assuming a minimum level of $0.5 \%$ of total protein). This is not surprising since episomal expression is known to be well below the levels derived from endogenous genes. Perhaps this is why transfection of GFP markers in mammalian cells typically uses either strong non-native viral promoters to drive expression (Tsien, 1998), or viral replication origins to amplify the plasmid to levels of up to $10^{4}$ copies per transfected cell (Kaufman, 1997). It is not clear whether this is required to produce the minimum amount of protein (between $10^{3}-10^{5}$ molecules) needed to detect fluorescence above background (Patterson et al., 1997; Tsien, 1998; Mazo et al., 1999) or because of the instability of the transformation. However, in the case of the episomal calmodulin promoter in Paramecium, we have demonstrated that it is easy to achieve protein levels substantially higher than the minimum number $\left(\sim 10^{7}\right.$ molecules $)$ required for detection (Fig. 2), which is maintained for at least 18 to 20 fissions.

Clearly, the ability of using a molecule like GFPmut1 not only for detection of transformation and expression, but also as a tag on specific molecules for monitoring physiological processes by in situ or in vivo studies, will be very important for future studies. Furthermore, GFPmut1 represents a FACS (fluorescence activated cell sorting)-optimized mutant GFP molecule (Cormack et al., 1996) and might be used to sort transformed populations of Paramecium. In conclusion, the establishment of GFP expression in Paramecium provides a useful tool for investigating a variety of questions that previously could not be addressed in this and some other related organisms.

Acknowledgements. We thank G. G. Borisy and S. Limbach for the generous use of the Nikon fluorescence microscope and for technical assistance. We also thank N. Klauke for assistance with microinjection and Y. Saimi for critical reading of the manuscript. This study was supported by grants from the Deutsche Forschungsgemeinschaft, grants "Ki678/1-1" and Forschergruppe "Struktur und Funktionssteuerung an zellulären Oberflächen" (project P4), as well as by the grant from National Institutes of Health, "GM 22714".

\section{References}

Berger, J. D. (1986): Autogamy in Paramecium: cell cycle stage-specific commitment to meiosis. Exp. Cell Res. 166, 475 - 485.

Boileau, A. J., Kissmehl, R., Kanabrocki, J. A., Saimi, Y. (1999): Transformation of Paramecium tetraurelia by electroporation or particle bombardment. J. Euk. Microbiol. 46, 56-65.

Chalfie, M., Tu, Y., Euskirchen, G., Ward, W. W., Prasher, D. C. (1994): Green fluorescent protein as a marker for gene expression. Science 263, $802-805$.

Cody, C. W., Prasher, D. C., Westler, W. M., Prendergast, F. G., Ward, W. W. (1993): Chemical structure of the hexapeptide chromophore of Aequorea green-fluorescent protein. Biochemistry 32, 1212 1218.

Cormack, B. P., Valdivia, R., Falkow, S. (1996): FACS-optimized mutants of the green fluorescent protein (GFP). Gene 173, 33-38.

Cubitt, A. B., Heim, R., Adams, S. R., Boyd, A. E., Gross, L. A., Tsien, R. Y. (1995): Understanding, improving and using green fluorescent proteins. Trends Biochem. Sci. 20, 448-455.

Elwess, N. L., Van Houten, J. L. (1997): Cloning and molecular analysis of the plasma membrane $\mathrm{Ca}^{2+}$-ATPase gene in Paramecium tetraurelia. J. Euk. Microbiol. 44, 250-257. 
Erxleben, C., Klauke, N., Flötenmeyer, M., Blanchard, M.-P., Braun, C., Plattner, H. (1997): Microdomain $\mathrm{Ca}^{2+}$ activation during exocytosis in Paramecium cells. Superposition of local subplasmalemmal calcium store activation by local $\mathrm{Ca}^{2+}$ influx. J. Cell Biol. 136, $597-607$

Fraga, D., Yano, J., Reed, M. W., Chuang, R., Bell, W., Van Houten, J. L., Hinrichsen, R. (1998): Introducing antisense oligodeoxynucleotides into Paramecium via electroporation. J. Euk. Microbiol. 45, $582-588$.

Gilley, D., Preer, J. R., Jr., Aufderheide, K. J., Polisky, B. (1988): Autonomous replication and addition of telomerelike sequences to DNA microinjected into Paramecium tetraurelia macronuclei. Mol. Cell. Biol. 8, 4765-4772.

Godiska, R., Aufderheide, K. J., Gilley, D., Hendrie, P., Fitzwater, T., Preer, L. B., Polisky, B., Preer, J. R., Jr. (1987): Transformation of Paramecium by microinjection of a cloned serotype gene. Proc. Natl. Acad. Sci. USA 84, 7590-7594.

Haas, J., Park, E.-C., Seed, B. (1996): Codon usage limitation in the expression of HIV-1 envelope glycoprotein. Curr. Biol. 6, 315 - 324.

Hauser, K., Kissmehl, R., Linder, J., Schultz, J. E., Lottspeich, F., Plattner, H. (1997): Identification of isoforms of the exocytosissensitive phosphoprotein PP63/parafusin in Paramecium tetraurelia and demonstration of phosphoglucomutase activity. Biochem. J. 323, $289-296$.

Haynes, W. J., Ling, K.-Y., Saimi, Y., Kung, C. (1995): Induction of antibiotic resistance in Paramecium tetraurelia by the bacterial gene APH-3'-II. J. Euk. Microbiol. 42, 83-91.

Haynes, W. J., Ling, K.-Y., Saimi, Y., Kung, C. (1996): Toward cloning genes by complementation in Paramecium. J. Neurogenet. 11, 81-98.

Heim, R., Prasher, D. C., Tsien, R. Y. (1994): Wavelength mutations and posttranslational autoxidation of green fluorescent protein. Proc. Natl. Acad. Sci. USA 91, $12501-12504$.

Heim, R., Cubitt, A. B., Tsien, R. Y. (1995): Improved green fluorescence. Nature 373, 663-664.

Inouye, S., Tsuji, F. I. (1994): Aequorea green fluorescent protein: Expression of the gene and fluorescent characteristics of the recombinant protein. FEBS Lett. 341, 277-280.

Kanabrocki, J. A., Saimi, Y., Preston, R. R., Haynes, W. J., Kung, C, (1991): Efficient transformation of $\mathrm{cam}^{2}$, a behavioral mutant of Paramecium tetraurelia, with the calmodulin gene. Proc. Natl. Acad. Sci. USA 88, $10845-10849$.

Kaufman, R. J. (1997): Overview of vector design for mammalian gene expression. In: R.S. Tuan (ed.): Methods in Molecular Biology: Recombinant Gene Expression Protocols, Humana Press, Totowa, New Jersey, Vol. 62, pp. 287-300.

Kissmehl, R., Huber, S., Kottwitz, B., Hauser, K., Plattner, H. (1998): Subplasmalemmal Ca-stores in Paramecium tetraurelia . Identification and characterisation of a sarco(endo)plasmic reticulum-like
$\mathrm{Ca}^{2+}$-ATPase by phosphoenzyme intermediate formation and its inhibition by caffeine. Cell Calcium 24, 193 -203.

Lefort-Tran, M., Aufderheide, K., Pouphile, M., Rossignol, M., Beisson, J. (1981): Control of exocytotic processes: cytological and physiological studies of trichocyst mutants in Paramecium tetraurelia. J. Cell Biol. 88, $301-311$.

Li, X. Q., Zhao, X. H., Fang, Y., Jiang, X., Duong, T., Huang, C. C., Kain, S. R. (1998): Generation of destabilized green fluorescent protein transcription reporter. J. Biol. Chem. 273, 34970 - 34975.

Madeddu, L., Gautier, C., Vayssié, L., Houari, A., Sperling, L. (1995): A large multigene family codes for the polypeptides of the crystalline trichocyst matrix in Paramecium. Mol. Biol. Cell 6, 649-659.

Martindale, D. W. (1989): Codon usage in Tetrahymena and other ciliates. J. Protozool. 36, 29-34.

Mazo, I. A., Levy, J. P., Muldoon, R. R., Link, C. J., Jr., Kain, S. R. (1999): Retroviral expression of green fluorescent protein. Methods Enzymol. 302, 329-341

McCormick-Graham, M., Haynes, W. J., Romero, D. P. (1997): Variable telomeric repeat synthesis in Paramecium tetraurelia is consistent with misincorporation by telomerase. EMBO J. 16, 3233-3242.

Patterson, G. H., Knobel, S. M., Sharif, W. D., Kain, S. R., Piston, D. W. (1997): Use of the green fluorescent protein (GFP) and its mutants in quantitative fluorescence microscopy. Biophys. J. 73, 2782 - 2790.

Prasher, D. C., Eckenrode, V. K., Ward, W. W., Prendergast, F. G., Cormier, M. J. (1992): Primary structure of the Aequorea victoria green fluorescent protein. Gene 111, 229-233.

Preer, J. R., Jr., Preer, L. B., Rudman, B. M., Barnett, A. J. (1985): Deviation from the universal code shown by the gene for surface protein 51A in Paramecium. Nature 314, 188-190.

Prescott, D. M. (1994): The DNA of ciliated protozoa. Microbiol. Rev. 58, 233-267.

Sambrook, J., Fritsch, E. F., Maniatis, T. (1989): Molecular Cloning: A Laboratory Manual, Cold Spring Harbour Lab., Cold Spring Harbour, New York.

Schlichtherle, I. M., Roos, D. S., Van Houten, J. L. (1996): Cloning and molecular analysis of the bifunctional dihydrofolate reductase thymidylate synthase gene in the ciliated protozoan Paramecium tetraurelia. Gen. Genet. 250, 665-673.

Sonneborn, T. M. (1979): Methods in Paramecium research. In: D. M. Prescott (ed.): Methods of Cell Physiology, Academic Press, New York, Vol. 4, pp. 241-339.

Sonneborn, T. M. (1975): The Paramecium aurelia complex of 14 sibling species. Trans. Am. Microsc. Soc. 94, 155-178.

Tsien, R. Y. (1998): The green fluorescent protein. Annu. Rev. Biochem. 67, 509-544.

Yang, T. T, Cheng, L., Kain, S. R. (1996): Optimized codon usage and chromophore mutations provide enhanced sensitivity with the green fluorescent protein. Nucleic Acids Res. 24, 4592-4593. 